\title{
Outreach at Washington State University: a case study in costs and attendance
}

Elizabeth Bernhardt, Viktor Bollen, Thomas Bersano, Sean Mossman

Elizabeth A. Bernhardt, Viktor Bollen, Thomas M. Bersano, Sean M. Mossman, "Outreach at Washington State University: a case study in costs and attendance," Proc. SPIE 9946, Optics Education and Outreach IV, 99460C (27 September 2016); doi: 10.1117/12.2236719

SPIE Event: SPIE Optical Engineering + Applications, 2016, San Diego, California, United States 


\title{
Outreach at Washington State University: a case study in costs and attendance
}

\author{
Elizabeth A. Bernhardt, Viktor Bollen, Thomas M. Bersano, Sean M. Mossman \\ Department of Physics and Astronomy, Washington State University, \\ Pullman, WA, 99164-2814, USA;
}

\begin{abstract}
Making effective and efficient use of outreach resources can be difficult for student groups in smaller rural communities. Washington State University's OSA/SPIE student chapter desires well attended yet cost-effective ways to educate and inform the public. We designed outreach activities focused on three different funding levels: low upfront cost, moderate continuing costs, and high upfront cost with low continuing costs. By featuring our activities at well attended events, such as a pre-football game event, or by advertising a headlining activity, such as a laser maze, we take advantage of large crowds to create a relaxed learning atmosphere. Moreover, participants enjoy casual learning while waiting for a main event. Choosing a particular funding level and associating with well-attended events makes outreach easier. While there are still many challenges to outreach, such as motivating volunteers or designing outreach programs, we hope overcoming two large obstacles will lead to future outreach success.
\end{abstract}

Keywords: Outreach, effective outreach budgeting, outreach design, international year of light laser maze, low effort outreach advertising

\section{INTRODUCTION}

Washington State University, located in Pullman, Washington, sits in the midst of a large agricultural region known as the Palouse. The rural location limits the number of people reachable with an outreach program. This challenge requires creative use of outreach budgeting and limited volunteer manhours to reach the largest possible audience. To confront this problem, we took several years to grow our outreach through deliberate scaling of costs and increasing use of university resources.

In this paper, we present three activities demonstrating how our outreach program changed over time. Each activity is discussed in terms of its relative upfront cost, relative continuing costs, and use of university resources. Since volunteers are a precious limited resource, the number of manhours to run these events are counted. The first activity is a low upfront cost, low continuing cost polarization demonstration. The second activity is a low upfront cost, high continuing cost spectrometer activity designed for a summer camp. The final activity is a high upfront cost, low continuing cost laser maze which, to date, has been seen by over 1000 participants. Through these activities, we demonstrate how other groups could maximize a budget and attract participants with minimum effort.

\section{POLARIZATION DEMONSTRATION}

In the polarization demo, attendees of all ages are introduced to the idea of polarization, and they are shown the science behind a polariscope. The attendees first watch as two polarizing film sheets are rotated in front of a light source. Then other items, such as another polarizing sheet, various clear plastic products, or birefringent materials, are placed between the polarizers. Attendees are encouraged to test their sunglasses or phone screens to see if they are polarized.

The polarization demonstration falls into the class of demonstrations widely known as a table demo. The table demo is often defined by its short duration (less than 5 minutes) and portability. Instead, we present it as an example of a low up-front cost, low continuing cost demonstration. Because of their low cost, table demos are excellent for any group with a new or small outreach program or a great addition to an already established program.

Further author information: Send correspondence to Elizabeth A. Bernhardt: E-mail: eabernhardt@wsu.edu 


\subsection{Costs}

The polarization demo is a low up-front cost, low recurring cost demonstration. In the most basic version of the demo, three sheets of polarizing film, a light source, and various clear plastic products are needed. The sheets of polarizing film can be purchased for approximately 10USD. The light source can be as simple as an LED. Overhead projectors are useful for performing the demo for larger groups and are often accessible to students at a university. The obvious drawback of an overhead projector is the need for a source of power. In either case, the cost to the presenter is at most a few dollars. Many plastic products have been tested in this demonstration: utensils, bags, cd cases. Presenters are encouraged to try out other plastics. If necessary, these products can be purchased for a few dollars. Birefringent crystals also perform well under the polarized light.

The continuing costs of this demonstration are low. All the materials can be reused. One person can lead the demonstration. Therefore the only recurring cost is a number of manhours equal to the duration of the event where the demonstration is hosted.

\subsection{Participation}

A table demo can be set up anywhere but does not draw an audience on its own. Fortunately, universities organize several events a year where a table demo is appropriate. Our group has hosted table demos at student organization fairs, student poster sessions, and at our own laser maze (see sec. 4). Polarization was also introduced as a small part of a summer camp outreach program (see sec. 3.2). In each of these cases, the larger event was responsible for providing an audience for the polarization demo, and, due to our association with the university, we were allowed to host a table for free. For small demos, readers are encouraged to reach out to their university or town and request space at public events, such as fairs or community events. There are many event organizers who may be willing to provide a local student group with a demo table.

\section{SPECTROMETER ACTIVITY}

Handmade spectrometers are a fun way for students to learn about spectroscopy.* In this activity, participants aged 12 to 18 build their own spectrometer, observe various light sources, and take home the spectrometer when finished. First, volunteers discuss a basic atomic model with the students. Next, the students build their own spectrometers following volunteer-led instructions. Finally, students observe various light sources, such as lasers and atomic gas lamps, and record their observations using colored pencils.

The beginning of the activity generally includes a fill-in-the-blank handout discussing atoms. The discussion walks students through a simplified mass-and-spring model of atoms, setting up a knowledge base for how photons interact with atoms to form discrete atomic spectra. Next, students build a spectrometer using foam board, tape, a small diffraction grating, and a paper scale. Once assembled, observations begin. A wrap-up discussion asks students about their observations and what the observations mean.

\subsection{Costs}

Minimizing costs is generally very important for most outreach budgets. The spectrometer activity is an example of a low-upfront cost and low continuing cost activity. First, upfront costs are flexible and can be adjusted for any budget. Second, continuing costs are approximately $40 \%$ volunteer manhours, which generally do not impact an outreach budget but must be considered when recruiting outreach volunteers.

To ensure safety of participants, a significant amount of prep work must be done before the activity, and we include manhour costs as calculated from US minimum wage of 7.25USD per hour (see Table 1). Most of the prep work requires volunteers to cut foam board pieces for the spectrometers using box cutters. While this particular prep work does not necessarily cut into an outreach budget, a nontrivial amount of volunteer manhours could limit the number of times this activity can be performed. Cutting the foam board pieces takes approximately 15 minutes per participant. It may be tempting to allow particpants to cut their own spectrometers pieces; however, box cutters are very sharp and we highly recommend pre-cutting pieces to avoid accidents and injury. A small amount of time is usually devoted to gathering supplies, collecting light sources, and training volunteers.

\footnotetext{
*Instructions for this activity are available at http://wsu.osahost.org/outreach/demos-activities/ introduction-to-spectroscopy/.
} 
Low and flexible upfront costs means this particular activity can be catered to an outreach budget. There are very few mandatory upfront purchases. Should this activity be performed at a school or if the participants can provide their own outreach supplies, a boxcutter is the only mandatory upfront cost, totaling approximately 5.50USD. Since this activity was adjusted for a summer camp, purchasing office supplies are included in Table 2. Fortunately, many office supplies are readily available or can be purchased either in bulk or for low cost. This activity only requires one set of scissors to cut tape when students are assembling their spectrometers. Furthermore, upfront costs can be lowered by observing sources of light already available, such as the sun or lamps or lightbulbs in the space where the outreach takes place.

Continuing costs make up the majority of this activity's budget. Each student takes home the spectrometer after the activity, so new materials must be purchased each time the activity is performed. As can be seen in Table 1, approximately half of the continuing costs are materials. Fortunately, many of the materials may be purchased in bulk to minimize costs. The diffraction grating is purchased as a large sheet and cut into small pieces. Similarly, aluminum foil is cut into small strips. These strips are then folded by the students to form the slit to allow light into their spectrometer. The cost for foam board was based on past execution of this outreach and how many pieces we could cut from a particular piece of foam board. Tape cost was estimated based on the necessary amount of tape to construction the spectrometer, then half a meter added to the estimate to account for waste. This length was then used to calculate the cost per participant.

Should a larger upfront budget be available, there are many additional light sources or upgrades one could purchase. Perhaps the easiest and most cost effective upgrade is providing patterned duct tape for constructing the spectrometers. Fun patterns allows students to personalize their spectrometer. Including lasers, atomic sources, and various light bulbs adds to the educational value by having more spectra and more sources for the student to observe. Laser pointers of various colors can be found at very reasonable prices or purchased in bulk. Atomic emission spectra lamps may be purchased through education vendors; we utilize various atomic gas lamps from the introduction to astronomy courses through our school's physics and astronomy department. Borrowing these lamps saves funding for other uses, provided participants either watch volunteers handle the expensive equipment or are very careful while using them. Finally, another fun light source is various colored LEDs. Our club borrows a tower of LEDs from the physics and astronomy department demo supplies, but purchasing supplies and wiring up circuits to power LEDs is very easy.

Two additional suggested upgrades change the activity slightly or make the prep work much easier. The spectrometer activity was originally designed for a science class of 12 -year-olds. The class period was much too short to build a spectrometer and use it, so pre-made spectrometers were purchased from an educational supply company. Each spectrometer cost just under 7USD. While quite expensive, pre-made spectrometers give the activity flexibility in where the outreach is performed, and the pre-made spectrometers can be collected and reused. Next, cutting out the foam board pieces for building the spectrometers can be a tedious task. Should funding be available, a laser cutting machine could be built or purchased to cut the foam board. However, this could be quite an expensive purchase; one should consider how many manhours are put into preparing for this activity. For a group of 30 students, the equivalent manhours of prep-work is just over 54USD, assuming minimum wage of 7.25USD per hour.

\begin{tabular}{|c|c|}
\hline Item & Cost per participant (USD) \\
\hline Diffraction grating & 0.10 \\
Foam board & 1.50 \\
Tape & 0.52 \\
Aluminum foil & 0.01 \\
Prep work man hours & 1.82 \\
\hline Total & 3.95 \\
\hline
\end{tabular}

Table 1. Approximate continuing costs per participant for the Do-It-Yourself spectrometer activity. Costs are estimates based on online pricing from a popular online retailer. Manhours are estimates based on current US minimum wage of 7.25USD per hour. 


\begin{tabular}{|c|c|}
\hline Item & Upfront cost (USD) \\
\hline Box cutter & 5.50 \\
12 Rulers & 9.59 \\
Pack of 96 pencils & 5.99 \\
Scissors & 1.00 \\
\hline Total & 22.08 \\
\hline
\end{tabular}

Table 2. Approximate upfront costs for the Do-It-Yourself spectrometer activity. Costs are estimates based on online pricing from a popular online retailer. Should participants be able to provide their own office supplies, upfront costs may be lowered.

\begin{tabular}{|c|c|}
\hline Item & Optional cost \\
\hline Patterned duct tape & $\$$ \\
Lasers of various colors & $\$ \$$ \\
Atomic spectra line sources & $\$ \$ \$$ \\
Various light bulbs: halogen, LED, compact fluorescent, incandescent & $\$$ \\
Various colored LEDs & $\$$ \\
Pre-made classroom spectrometers & $\$ \$ \$$ \\
\hline
\end{tabular}

Table 3. Optional upgrades increase the cost of the activity, but can increase the educational value and enjoyment for participants. Key: \$: low cost, less than 5USD; $\$ \$$ : medium cost, less than 50USD; $\$ \$ \$$ : high cost, greater than 50USD

\subsection{Participation}

The do-it-yourself spectrometer activity was initially designed as a stand-alone activity to bring to classrooms, hence the suggestion of purchasing pre-made spectrometers. However, the science teacher with whom our group collaborated moved to a different school district and the new science teacher was not interested in continuing the collaboration. As such, the spectrometer activity was adjusted to fit the needs of a summer camp.

Cougar Quest is a yearly summer camp where students aged 12 to 18 stay one week at the WSU campus and take three "courses". Courses are proposed by graduate students or faculty, and selected courses are approved by Cougar Quest facilitators based on educational value, entertainment value, and past camper approval of the selected topic or similar topics. Each course has four - two hour periods to guide students through activities. As the goal of the Cougar Quest is encouraging students to enjoy learning, courses are discouraged from relying on lectures longer than 20 minutes. Since the spectrometer activity is very hands-on, it was naturally adapted to high energy campers.

First, the pre-made spectrometers utilized for classrooms were replaced with spectrometers the students build and take home. This proved to be the most enjoyable part of summer camp for most participants, as most science courses did not have items for the campers to bring home. Next, many light sources were purchased or borrowed to show the breadth of spectra, such as discrete sources or continuous sources. Students also observed fluorescence, but utilized small hand-held diffraction gratings because it was very difficult to align the spectrometer with the fluorescing light source.

Relying on a summer camp meant the club did not need to focus on attracting participants and could instead focus on designing entertaining, educational content. As Cougar Quest is an already established summer camp opportunity, the club simply had to write an interesting description to attract students to the course. Moreover, the spectrometer activity could not be stretched into an eight hour activity. Hence, a four part series on paradigms of optics was developed; each two hour period focused on a different paradigm. The spectrometer activity fit nicely into the "light as a particle" paradigm and was easily expanded to cover a two hour period. Taking advantage of an already organized summer camp attracted participants with very little effort on the club's part. Besides Cougar Quest, many other organizations in Pullman sponsor and organize summer camps, such as the YMCA, the Girl Scouts of America, and even our local farmers market. Readers are encouraged to reach out to local organization and inquire about summer camps, after school activities, or other time periods where the local organization can gather the participants and the reader can provide the educational outreach content. We find this symbiotic relationship allows each group to focus on what it does best. 


\section{LASER MAZE}

The laser maze is an implementation of the classic heist movie scenario where a thief maneuvers through a room filled with lasers without breaking the laser beams for fear of triggering an alarm. Recreating this activity required only three low powered lasers linked to detectors so that when a beam is broken an alarm would go off. By using mirrors on adjustable mounts, fine control of the beam's direction allows adjustment of the difficulty of the maze. As an important note, it is easy to over design a laser maze; we keep our presented design simple and suggest fancy features for later iterations of the maze. ${ }^{\dagger}$

\subsection{Prep}

The biggest time investment for this event is building all the components for the laser maze, such as the electronics, software, and mounts. Preparations occurred for the WSU laser maze over the course of several months, since there was a nontrivial amount of prototyping involved.

On the day of the event, setting up the electronics and lasers only takes about an hour for two to three people. In theory, the setup could be done by one person, but at least two is recommended. In addition to the obvious parts of the maze (lasers, fog machine, and alarms), many safety measures are put in place and the event space is darkened. The more time consuming work is setting up black out curtains and decorating the maze with fluorescent features. In total, prep work takes approximately five manhours.

\subsection{Cost}

We received an SPIE International Year of Light Student Outreach activity grant for this project. Since this was our club's first attempt at a large-scale project, keeping costs low and sticking to the proposed budget was very important during development.

Designing a laser maze requires many items: lasers, a fog machine, and black-out curtains. Inexpensive $5 \mathrm{~mW}$ green laser pointers were chosen because the $5 \mathrm{~mW}$ power puts the laser pointers into laser safety class $3 \mathrm{R}$, the highest class one can go before needing safety equipment. It is important to note a high power laser and many mirrors could be used to create a maze, but safety concerns, such as blinding participants, should be a priority for any group considering building a laser maze. The $5 \mathrm{~mW}$ laser pointers were disassembled and attached to an external power supply and controller. During testing, it was discovered red laser diodes were not as visible as the green laser pointers, so the red lasers were abandoned. To increase laser visibility, a water-based fog machine was utilized. While an oil-based fog machine may be less expensive, the water-based machines are less likely to agitate attendants who have asthma or lung problems. The machine was rather expensive, but it is vital for seeing the beams. However, the fog machine's efficiency means recurring costs are limited; the fog machine is still running on its original water-based cartridge. Additionally, the event venue had a lot of unwanted light, so large, fireproof black fabric curtains were made and hung on a custom-built PVC frame. The dimly lit room, combined with the fog, makes the lasers very visible.

Several design choices were made to give flexibility and freedom in setting up the maze while maintaining safety and staying on budget. First, the deconstructed lasers were mounted in custom rigid holders so that they would not move or drift over time. Instead of purchasing many lasers and sending each beam from the laser straight to a detector, the beam was bounced off a few mirrors before being detected. The chosen lasers could be bounced off of three mirrors before the beam was too dirty to be detected by the chosen photodetectors. To mount the mirrors and detectors, small ball joint mounts were made out of PVC and attached to the wall using adhesive poster strips. The ball joint allows angling of the mirrors to aim the laser beams more precisely. The flexibility in setting up the maze allows us to adapt it to various venues of different sizes and makes it very mobile.

Next, photoresistors served as the detector for the laser beam being broken, but they needed to be paired with a laser beam. During setup, an analog signal was read from the photoresistor, and each photoresistor was paired with a laser beam. This pairing was created by first reading the signal from one photoresistor, then cycling through all the lasers, turning each on and off, pairing the photoresistor with the laser for which the

${ }^{\dagger}$ The authors encourage readers to use this paper as a guide to design their own maze. The code and maze design are not publicly available. 
detector had the highest response. Creating the pairing during setup allows much more freedom in the design of the maze and speeds up setup time.

Arduino microcontrollers were chosen to control the entire maze due to ease in programming and connecting hardware. To connect the lasers and detectors to the Arduino, Cat-5 Ethernet cables were purchased. Each cable has eight wires, allowing connection to four devices, or two laser-detector pairs. This option was less expensive than buying bulk raw wire of equal length, and Ethernet plugs and jacks connected the cables to the microcontroller. Since the laser could only be bounced off three mirrors before decoherence interfered with the detectors, the lasers and detectors could be placed on the same side of the room, removing any tripping hazards from wires going across the room.

To interface with the controller, a custom Python program was created that runs on a laptop. Python was chosen because all the needed software packages are free, and documentation is readily available.

The hardware and software constituent the majority of the upfront costs for the maze, while disposable items and small prizes comprise the recurring costs. First, the poster strips must be purchased for each setup of the maze. A prize at the end of the maze for participants is the next recurring cost; candy, stickers, or other small prizes were popular during the various iterations of this event. Decorations are optional and may be disposable or reusable. While volunteer manhours do not impact most budgets, setup takes about two to three manhours (assuming no decorations). During our events, we find it is necessary to have one person ensuring working electronics while stopping participants from touching the mounts, one person refilling the prize bowl while running the fog machine, and one person giving instructions while letting participants in and out of the maze. Costs are summarized in Table 4.2.

\begin{tabular}{|c|c|}
\hline Item & Approximate Cost (USD) \\
\hline Electronics & 400 \\
Blackout Curtains & 200 \\
\hline Initial Total & 600 \\
Recurring Cost & 30 \\
\hline
\end{tabular}

Table 4. Many hardware items are purchased for the laser maze activity, and these items can be generally separated into three groups: electronics, blackout curtains, and recurring costs.

\subsection{Participation}

Depending on the size of the room, the laser maze accommodates a few people at the same time. However, the participants should have plenty of space to move without running into each other. With our designed setup, the "thieves" move through the maze to the treasure and then escape back through the maze the way they entered. A time limit was implemented to keep participants from dilly-dallying inside the maze.

For the grand unveiling of the maze, the club desired to attract many participants. A significant amount of funding purchased various advertisements(see Table 4.3, while a large amount of manhours went into small outreach events to inform the public about the laser maze event. Most of our advertisement involved going to local elementary schools the month prior to the event and doing optics outreach for classes. Afterwards, we would tell the students about the laser maze and hand out a flier to take home. This advertisement option was the most effective, but also the most time intensive. The only cost was printing costs for the flier and volunteer time. We discovered the required manhours for the outreach-related advertisements, while extremely effective, could not be justified for future iterations of the maze. To judge the effectiveness of the different advertisement approaches, attendees filled out a questionnaire after they were done in the maze.

To maximize attendance, the event was scheduled during Mom's weekend at Washington State University, so that we could tap into the already activity filled day. This also allowed utilization of a variety of free advertisement channels on campus, such as digital and paper posters. The student run radio station allows free public awareness campaigns, so a short message about the laser maze was written. Additionally, an advertisement in the local community update paper was purchased. This was the most expensive option with the lowest return on investment. Overall, the grand unveiling attracted over 700 participants. 


\begin{tabular}{|c|c|}
\hline Advertisement & Cost (USD) \\
\hline Printer Ink (black) & 40 \\
Printer Ink (tri-color) & 35 \\
Copies & 12 \\
Community Update Ad & 160 \\
\hline Total & 247 \\
\hline
\end{tabular}

Table 5. Approximate costs for the different advertisements we used to inform the community about the laser maze. Many free avenues for advertisements were utilized as well.

During the second iteration of the maze the next year, our club focused advertising efforts to minimize required manhours. The small outreach sessions had to be cut due to lack of volunteers. However, many free avenues were still available. Univeristy resources and websites informed Mom's weekend participants of our event. Although attendance was significantly lower than the grand unveiling, approximately 300 participants attended the maze.

The most important part of the laser maze event is not the maze itself, but the excitement it attracts. Our club capitalized on the laser maze's popularity to perform additional outreach. First, a short demonstration on fiber optics and the internet allowed for crowd control while informing participants about optics and photonics. Second, the queue was staged right next to several hands-on demonstrations, such as a spectroscopy station and the polarization activity (see Section 2). Readers are encouraged to seek out popular events and set up a small table to perform outreach. For example, the geology department partnered with the laser maze event; they provided a small geology outreach table and we attracted participants. Taking advantage of already popular and well attended events allows outreach to reach many participants with little advertising effort.

\section{CONCLUSION}

In this paper we examined three different outreach activities performed at Washington State University with various upfront and continuing costs: a polarization demonstration, spectrometer building activity, and a laser maze. Additionally, each activity can be utilized in conjunction with other events to minimize advertising costs but increase participation.

The polarization demonstration is a low up front cost activity that can be run by just one person. Since one only needs to purchase the different polarizers and light sources once, the only continuing cost is manpower. This demonstration can easily be scaled from tabletop to classroom. By bringing this demonstration to fairs, large events, or other planned activities, participants naturally filter from the main event to the polarization demonstration.

The next step up in cost is the spectrometer activity, which has a low up front cost, since one only needs basic office supplies and boxcutter, but a high continuing cost for spectrometer construction materials. This small group to classroom sized activity is especially enjoyable because the participants are able to take the spectrometers with them afterwards. The spectrometer activity easily lends itself to summer camps or a classroom activity.

The laser maze's construction gives it the greatest upfront investment both in cost and labor, but a low continuing cost. As always, the greatest reoccurring expense is that of manhours. This event is also a great way to expose participants to other demonstrations by accompanying a large activity like the maze with smaller tabletop demonstrations. The flexibility built into the design of the maze allows us to adapt it to various venues, easily set the difficultly level of the maze, and attract participants to partake in other, smaller outreach demonstrations before enjoying the maze.

We hope that by examining the various costs of these activities we've shed some light as to what is possible in your area. Additionally, we hope to inspire new ways to attract participants to your outreach event with little advertising costs through symbiotic relationships with other groups in your area. 


\section{ACKNOWLEDGMENTS}

We thank SPIE's International Year of Light outreach grant for funding of development and implementation of the Laser Maze. Funding for other outreach activities include OSA student chapter grants, SPIE student chapter activity funding, WSU Graduate and Professional Student Association (GPSA) affiliate funding, and the WSU department of physics and astronomy. Successful outreach needs many manhours and the authors wish to acknowledge the contributions of WSU's OSA-SPIE student chapter members, especially Michele Moore, Maren Mossman, and Nate Turner. Our student chapter is deeply thankful for assistance from the members of the physics department, particularly Kris Boreen, Tom Johnson, and Tim Whitacre. Finally, we wish to thank our chapter's advisers, Mark G. Kuzyk and Robin Stratton. 